\title{
Dual matrix inequalities in stability and performance analysis of linear differential/difference inclusions
}

\author{
Rafal Goebel, Tingshu Hu, and Andrew R. Teel \\ Center for Control Engineering and Computation, Electrical and Computer \\ Engineering, University of California, Santa Barbara, CA 93106 \\ rafal, tingshu, teel@ece.ucsb.edu
}

Summary. The paper provides numerical examples to illustrate the recent results by the authors relating asymptotic stability and dissipativity of a linear differential or difference inclusion to these properties for the corresponding dual linear differential or difference inclusion. It is shown how this duality theory broadens the applicability of numerical algorithms for stability and performance analysis that have appeared previously in the literature.

\section{Introduction}

Perhaps the simplest pair of dual linear matrix inequalities for control systems is

$$
A^{T} P+P A<0,
$$

for a given matrix $A$ and a symmetric and positive definite $P$, and

$$
A Q+A^{T} Q<0,
$$

for a symmetric and positive definite $Q$. The two inequalities are equivalent through $Q=P^{-1}$, and then each of them characterizes the stability of both the linear system $\dot{x}(t)=A x(t)$ and its dual system $\dot{x}(t)=A^{T} x(t)$. Another pair of dual matrix inequalities is

$$
\left[\begin{array}{cc}
A^{T} P+P A+C^{T} C & P B \\
B^{T} P & -\gamma^{2} I
\end{array}\right]<0
$$

and

$$
\left[\begin{array}{cc}
A Q+Q A^{T}+B B^{T} & Q C^{T} \\
C Q & -\gamma^{2} I
\end{array}\right]<0 .
$$

They are equivalent through $Q=\gamma^{2} P^{-1}$, and then each characterizes the finite $L^{2}$-gain, bounded by $\gamma$, for the pair of dual systems $(A, B, C)$ and 
$\left(A^{T}, C^{T}, B^{T}\right)$. Further examples of pairs of dual matrix inequalities come from characterizing other input-output performances of linear systems, such as passivity or the Hankel norm.

The stated matrix inequalities arise from stability and performance analysis of linear systems through quadratic Lyapunov or storage functions. The use of such functions in the analysis of differential inclusions, leading to matrix inequalities like these in (1) to (4) but holding for all $A$ (or all $A, B, C$ ) in a certain set, is possible; see [3].

While quadratic Lyapunov functions lead to easily tractable linear matrix inequalities and simplify computational issues a great deal, it has been realized that they can yield conservative evaluation of stability for linear differential inclusions (see, e.g., $[3,5,6,14,16,23])$. It is now well established that convex homogeneous - but not necessarily quadratic - Lyapunov functions are sufficient to characterize the stability of linear differential/difference inclusions (LDIs); see $[15,6]$. Recent years have witnessed an extensive search for homogeneous Lyapunov functions. Particular types of functions looked at are: piecewise quadratic Lyapunov functions $([15,22])$, polyhedral Lyapunov functions $([2,4])$, and homogeneous polynomial Lyapunov functions $([5,14,23])$.

Recently, more attention was given in $([7,13,11,10])$ to two particular classes of convex homogeneous Lyapunov functions: the functions given as the pointwise maximum of a family of quadratic functions, and those given as the convex hull of a family of quadratic functions. For simplicity, we refer to functions in these classes as max functions and convex hull functions. It is shown with an example in [3] (page 73) that a max function may validate the stability of an LDI even when all quadratics fail. The convex hull function was first used for stability purposes in $[11,10]$ to estimate the domain of attraction for saturated linear systems and systems with a generalized sector condition (where it was called a "composite quadratic" function). Finally, $[7,13]$ noted and explored the convex duality between the two classes, and used it to enhance stability analysis of LDIs and saturated linear systems.

Furthermore, [7] established a set of important symmetric relationships between dual linear differential inclusions. Stability of the LDI ${ }^{1}$

$$
\dot{x}(t) \in \operatorname{co}\left\{A_{i}\right\}_{i=1}^{m} x(t)
$$

was shown to be equivalent to stability of the dual LDI

$$
\dot{\xi}(t) \in \operatorname{co}\left\{A_{i}^{T}\right\}_{i=1}^{m} \xi(t) .
$$

Based on the max function and the convex hull function, the following matrix inequalities are suggested:

$$
A_{i}^{T} P_{j}+P_{j} A_{i} \leq \sum_{k=1}^{l} \lambda_{i j k}\left(P_{k}-P_{j}\right)-\gamma P_{j}
$$

\footnotetext{
${ }^{1}$ The LDI means that $\dot{x}(t)$ is an element of the convex hull of points $A_{i} x(t)$, $i=1,2, \ldots, m$, for almost all $t$.
} 
for all $i=1,2, \ldots m, j=1,2, . . l$ and

$$
Q_{j} A_{i}^{T}+A_{i} Q_{j} \leq \sum_{k=1}^{l} \lambda_{i j k}\left(Q_{k}-Q_{j}\right)-\gamma Q_{j}
$$

for all $i=1,2, \ldots m, j=1,2, . . l$. If there exist positive definite and symmetric matrices $P_{1}, P_{2}, \ldots, P_{l}$ and nonnegative numbers $\lambda_{i j k}$ solving (7) or matrices $Q_{1}, Q_{2}, \ldots Q_{l}$ and numbers $\lambda_{i j k} \geq 0$ solving (8), then both (5) and (6) are stable, similarly to what occurs for linear systems.

However, the matrix inequalities (7) and (8) are no longer equivalent. This might be unexpected but can be explained since either (7) or (8) is only a sufficient condition for stability of the LDIs. For linear systems, existence of a solution to (1) or (2) is sufficient, but also necessary for stability.

Even though the dual matrix inequalities (7) and (8) may still be conservative for stability analysis, numerical examples have shown that they may significantly improve on what can be achieved by quadratics. Furthermore, by duality, we can combine (7) and (8) to obtain a better estimate: if either inequality is satisfied, then the stability of both LDIs is confirmed.

Similar matrix inequalities can be stated to evaluate the $L^{2}$-gain, dissipativity, and other input-output performance measures for LDIs. We give several examples in this paper. As can be seen from (7) and (8), the matrix inequalities based on max functions or convex hull functions are bound to be more complicated than their counterparts derived from quadratic functions, as a price for reducing conservatism. In fact, (7) and (8) are bilinear matrix inequalities (BMIs) instead of LMIs. Despite the well-known fact that BMIs are NP-hard, attempts have been made to make them more tractable; see, e.g., $[1,8,9]$. By using the path-following method presented in [9], we have developed a handful of algorithms to solve (7), (8) and other dual matrix inequalities arising from performance analysis. Our numerical experience shows that the path-following method is very effective. (We used straightforward iterative schemes in our earlier computation without much success.)

The purpose of this paper is to present several matrix inequalities, some of which were previously stated in [7], for stability and performance analysis of LDIs, and illustrate their applicability and effectiveness by way of examples. Through this, we also justify the application of convex duality theory, and motivate the interest in the class of convex functions given by a maximum of quadratics or the functions given by a convex hull of quadratics.

The material is organized as follows. Section 2 is the theoretical one, and outlines how the use of convex conjugate functions leads to a duality theory for LDIs; the details are in [7]. The two classes of Lyapunov and storage functions that our numerical tools are based on are described in Section 3. Also there, various examples related to stability, and relying on (7) and (8) are given. For further details, see [7, 13]. A general reference for convex analysis materials we use is [17]; see also [18]. In Section 4, we discuss examples related to dissipativity properties of LDIs, more specifically the $L^{2}$-gain and passivity. 
General results for such properties are the topic of a forthcoming work by the authors; for dissipativity concepts for linear systems, consult [20], [21]. Section 5 focuses on discrete-time systems, and includes an example of an application of the matrix inequalities technology to estimation of domains of attraction of nonlinear systems (for details on the continuous-time case, see [13]).

\section{Duality of Lyapunov functions}

In the analysis of linear differential and difference inclusions, in contrast to equations, relying on quadratic Lyapunov functions is not sufficient. For example, the linear differential inclusion (5) can be asymptotically (and then in fact exponentially) stable when no quadratic Lyapunov function exists, i.e. when for no symmetric and positive definite $P$ we have $A_{i}^{T} P+P A_{i}<0$ for $i=1,2, . ., m .{ }^{2}$ Existence of such a matrix leads to stronger stability of (5), called quadratic stability. See Example 2. Consequently, in order to establish a duality theory for linear differential and difference inclusions, for example results stating that (5) is asymptotically stable if and only if (6) is asymptotically stable, one may need to look at Lyapunov functions that are not quadratic, and moreover, to find a relationship for such functions corresponding to that between $P$ and $P^{-1}$ for quadratic ones.

If (5) is asymptotically stable, then there exists $\gamma>0$ and a differentiable, strictly convex, positive definite, and homogeneous of degree 2 function $V$ such that

$$
\nabla V(x)^{T} A x \leq-\gamma V(x) \text { for all } x,
$$

for all $A \in \operatorname{co}\left\{A_{i}\right\}_{i=1}^{m} \cdot{ }^{3}$ Homogeneity of degree 2 of $V$ means that for all $\lambda \in \mathbb{R}, V(\lambda x)=\lambda^{2} V(x)$. In what follows, we will denote by $\mathcal{L}$ the class of all differentiable, strictly convex, positive definite, and homogeneous of degree 2 functions. ${ }^{4}$ Of course, every quadratic function $\frac{1}{2} x^{T} P x$ with a symmetric and positive definite $P$ is in $\mathcal{L}$.

It turns out that the convex functions of class $\mathcal{L}$ are very well suited to support a duality theory for linear differential and difference inclusions. Moreover, the key construction, leading from one such convex function to another and reflecting the relationship between $P$ and $P^{-1}$ for quadratic Lyapunov

${ }^{2}$ The Lyapunov inequality holding at each of $A_{i}$ 's is sufficient for it to hold at each element of $\operatorname{co}\left\{A_{i}\right\}_{i=1}^{n}$.

${ }^{3}$ The inequality (9) corresponds to the bound $\|x(t)\| \leq c\|x(0)\| e^{-\frac{1}{2} \gamma t}$ on solutions of the LDI. Slightly abusing the notation, we will refer to $\gamma$ as the convergence rate, or the constant of exponential stability.

${ }^{4}$ Asymptotic stability of (5) is equivalent to exponential stability. If (5) is exponentially stable with a coefficient $\bar{\gamma} / 2>0$, then $\gamma$ in (9) can be chosen arbitrarily close, but smaller than, $\bar{\gamma}$. If one does not insist on differentiability of $V$, a Lyapunov inequality with $\bar{\gamma}$ can be written, using the convex subdifferential of $V$ in place of $\nabla V$. 
functions, exists and is well appreciated in convex analysis and optimization. It is the construction of a convex conjugate function. For any $V \in \mathcal{L}$, its convex conjugate is defined as

$$
V^{*}(\xi)=\sup _{x}\left\{\xi^{T} x-V(x)\right\} .
$$

The function $V^{*}$ is also convex, and in fact $V^{*} \in \mathcal{L}$. For example, verifying that it is homogeneous can be done directly: for any $\lambda \neq 0$, we have

$$
\begin{aligned}
V^{*}(\lambda \xi) & =\sup _{x}\left\{(\lambda \xi)^{T} x-V(x)\right\}=\lambda^{2} \sup _{x}\left\{\frac{1}{\lambda} \xi^{T} x-\frac{1}{\lambda^{2}} V(x)\right\} \\
& =\lambda^{2} \sup _{x}\left\{\xi^{T}\left(\frac{x}{\lambda}\right)-V\left(\frac{x}{\lambda}\right)\right\}=\lambda^{2} \sup _{x}\left\{\xi^{T} x-V(x)\right\} .
\end{aligned}
$$

The last expression is exactly $\lambda^{2} V^{*}(\xi)$. Showing the other properties of $V^{*}$ requires more elaborate techniques. ${ }^{5}$ The relationship between $V$ and $V^{*}$ is one-to-one, and in fact it is symmetric: the conjugate of $V^{*}$ is $V$ :

$$
\left(V^{*}\right)^{*}(x)=\sup _{\xi}\left\{x^{T} \xi-V^{*}(\xi)\right\}=V(x)
$$

In particular, $V \in \mathcal{L}$ if and only if $V^{*} \in \mathcal{L}$. This, in a sense, generalizes the fact that $P$ is symmetric and positive definite if and only if $P^{-1}$ is.

Even more importantly, the convex conjugate turns out to be exactly the object one needs when passing from a Lyapunov inequality verifying stability of a linear differential or difference inclusion to a Lyapunov function verifying the stability of a dual inclusion. This is particularly striking for difference inclusions; nothing more than the very definition of $V^{*}$ is then needed. Indeed, suppose that for some $V \in \mathcal{L}$ and some $\gamma \in(0,1)$ we have

$$
V(A x) \leq \gamma V(x) \text { for all } x .
$$

We will now show that this condition is equivalent to

$$
V^{*}\left(A^{T} \xi\right) \leq \gamma V^{*}(\xi) \text { for all } \xi
$$

The argument is a direct computation. Suppose that (12) is true. Then

$$
V^{*}\left(A^{T} \xi\right)=\sup _{x}\left\{\left(A^{T} \xi\right)^{T} x-V(x)\right\} \leq \sup _{x}\left\{\xi^{T} A x-\frac{1}{\gamma} V(A x)\right\},
$$

where the inequality comes from replacing $V(x)$ by the bound coming from (12). The expression on the right above can be rewritten as a supremum taken

${ }^{5}$ In general, for finite convex functions, conjugacy gives an equivalence between strict convexity of a function and differentiability of its conjugate, and vice versa. Thus, the conjugate of a strictly convex and differentiable function is differentiable and strictly convex. 
over the range of $A$, which is not greater than the supremum taken over the whole space. That is,

$$
V^{*}\left(A^{T} \xi\right) \leq \frac{1}{\gamma} \sup _{z \in \operatorname{rge} A}\left\{(\gamma \xi)^{T} z-V(z)\right\} \leq \frac{1}{\gamma} \sup _{x}\left\{(\gamma \xi)^{T} x-V(x)\right\} .
$$

The supremum on the right is exactly $V^{*}(\gamma \xi)$. As we already know that $V^{*}$ is positively homogeneous of degree 2 , we obtain $V^{*}\left(A^{T} \xi\right) \leq \gamma V^{*}(\xi)$, which is the inequality in (13).

An immediate and important consequence of the fact that a Lyapunov inequality (12) translates to the dual inequality (13) is that stability of a linear difference inclusion is equivalent to stability of its dual. More specifically,

(i) the linear difference inclusion ${ }^{6}$

$$
x^{+} \in \operatorname{co}\left\{A_{i}\right\}_{i=1}^{m} x
$$

is exponentially stable with constant $\gamma$

if and only if

(ii) the dual linear difference inclusion

$$
\xi^{+} \in \operatorname{co}\left\{A_{i}^{T}\right\}_{i=1}^{m} \xi
$$

is exponentially stable with constant $\gamma$.

A corresponding result for continuous time relies on the relationship between $\nabla V$ and $\nabla V^{*}$. These mappings are inverses of one another; further refinement of that fact for positively homogeneous functions leads to the desired result: a function $V \in \mathcal{L}$ is such that the Lyapunov inequality (9) holds if and only if $V^{*} \in \mathcal{L}$ is such that the dual Lyapunov inequality,

$$
\nabla V^{*}(\xi)^{T} A^{T} \xi \leq-\gamma V^{*}(\xi) \text { for all } \xi
$$

is satisfied. Consequently, as in discrete time, we have:

(i) the linear differential inclusion (5) is exponentially stable with constant $\gamma$ if and only if

(ii) the dual linear differential inclusion (6) is exponentially stable with constant $\gamma$.

An immediate benefit of the equivalence of stability, for both differential and difference inclusions, is that it doubles the number of numerical tools one can use to establish stability. Given a particular numerical technique to test whether a differential (or difference) inclusion given by $A_{1}, A_{2}, \ldots, A_{m}$ is

\footnotetext{
${ }^{6}$ The linear difference inclusion (14) means that $x(k+1)$ is an element of the convex hull of points $A_{i} x(k), i=1,2, \ldots, m$, for all $k$.
} 
asymptotically stable, one can apply it as well to the transposes. If either test - for the original matrices or for the transposes - shows stability, the theory we described concludes that in fact both inclusions - the original one and the dual one - are stable. This simple trick can lead to very surprising results.

Example 1. Consider

$$
A_{1}=\left[\begin{array}{ccc}
0 & 1 & 0 \\
0 & 0 & 1 \\
-1 & -2 & -4
\end{array}\right], M=\left[\begin{array}{ccc}
-2 & 0 & -1 \\
1 & -10 & 3 \\
3 & -4 & 2
\end{array}\right], A_{2}=A_{1}+a M
$$

with $a>0$. Then consider the LDI with the state matrix belonging to the set $\operatorname{co}\left\{A_{1}, A_{2}(a)\right\}$. One of the previously used numerical tests for stability of LDIs, proposed in [5], searches for the existence of a homogeneous polynomial Lyapunov function (HPLF). This test, when applied using fourth-order HPLFs to $A_{1}, A_{2}(a)$, shows that the LDI is stable for positive $a$ up to 75.1071. That same test, applied to $A_{1}^{T}, A_{2}^{T}(a)$, and thus testing the stability of an LDI given by $\operatorname{co}\left\{A_{1}^{T}, A_{2}(a)^{T}\right\}$, shows it is stable for all positive $a$ ! By duality, the original inclusion is also stable for all such $a$ 's.

Another benefit is that duality helps in designing numerical tests and identifying favorable classes of potential Lyapunov functions. We illustrate this in the next section.

\section{Classes of potential Lyapunov functions}

A linear differential inclusion (5) is asymptotically, and then in fact exponentially stable if and only if there exists a convex, positive definite, and homogeneous of degree 2 function $V$ such that the Lyapunov inequality (9) holds for all $A \in \operatorname{co}\left\{A_{i}\right\}_{i=1}^{m} .{ }^{7}$ To construct numerical tools that search for functions verifying stability of a given LDI, one needs to restrict attention to particular classes of potential Lyapunov functions. Here, we discuss two such classes, general enough to approximate any convex, positive definite, and homogeneous of degree 2 function, while also amenable to numerical methods.

First we note that, as it could be expected from the discussion at the beginning of Section 2, for quadratic functions we have

$$
V(x)=\frac{1}{2} x^{T} P x, \quad V^{*}(\xi)=\frac{1}{2} \xi^{T} P^{-1} \xi,
$$

\footnotetext{
${ }^{7}$ From now on, we do not insist that $V$ be differentiable. Consequently, inequality (9) should be understood to hold at all points where $V$ is differentiable, which is almost everywhere. This is in fact equivalent to (9) being valid with $\nabla V$ replaced by the subdifferential of $V$ in the sense of convex analysis. The subdifferential may be a set, and then (9) is understood to hold for every element of the subdifferential.
} 
when $P$ is symmetric and positive definite. It can be verified by a direct computation. Thus, quadratic functions given by symmetric and positive definite matrices form a class that is conjugate to itself.

The two classes of convex functions we want to use in stability analysis are conjugate to one another. They are: the functions given by a pointwise maximum of a family of quadratic functions and the functions given as the convex hull of a family of quadratic functions. More specifically, given symmetric and positive definite $P_{1}, P_{2}, \ldots, P_{l}$, by the max function we mean the pointwise maximum of the quadratic functions given by $P_{j}$ 's, that is

$$
V_{\max }(x)=\max _{j=1,2, . ., l} \frac{1}{2} x^{T} P_{j} x .
$$

This function is strictly convex, positive definite, and homogeneous of degree 2 .

With the help of the max function, and also the convex hull function we discuss later, convenient bilinear matrix inequality conditions for stability can be obtained. For example, suppose we want to verify the stability of an LDI given by two matrices $A_{1}, A_{2}$, using the maximum of two quadratic functions. That is, we want to find $P_{1}, P_{2}$ such that $V_{\max }$ given by (18) is a Lyapunov function for the LDI under discussion. Pick a point $x$ where $V_{\max }$ is differentiable, and suppose that $\frac{1}{2} x^{T} P_{1} x \geq \frac{1}{2} x^{T} P_{2} x$. Then $\nabla V_{\max }(x)=P_{1} x$, and for $V_{\max }$ to satisfy (9) at $x$, we need

$$
x^{T}\left(P_{1} A_{i}^{T}+A_{i} P_{1}\right) x \leq-\gamma x^{T} P_{1} x
$$

for $i=1,2$. On the other hand, we do not need $x^{T}\left(P_{2} A_{i}^{T}+A_{i} P_{2}\right) x$ to be negative. Symmetrically, at points where $\frac{1}{2} x^{T} P_{2} x \geq \frac{1}{2} x^{T} P_{1} x$, we need

$$
x^{T}\left(P_{2} A_{i}^{T}+A_{i} P_{2}\right) x \leq-\gamma x^{T} P_{2} x
$$

for $i=1,2$. This suggests the following sufficient condition for $V_{\max }$ given by $P_{1}, P_{2}$ to be a Lyapunov function for $\dot{x}(t) \in \operatorname{co}\left\{A_{1}, A_{2}\right\} \mathrm{x}(\mathrm{t})$ : for some nonnegative $\lambda_{1}, \lambda_{2}, \lambda_{3}, \lambda_{4}$,

$$
\begin{aligned}
& P_{1} A_{1}^{T}+A_{1} P_{1} \leq \lambda_{1}\left(P_{2}-P_{1}\right)-\gamma P_{1}, \\
& P_{1} A_{2}^{T}+A_{2} P_{1} \leq \lambda_{2}\left(P_{2}-P_{1}\right)-\gamma P_{1}, \\
& P_{2} A_{1}^{T}+A_{1} P_{2} \leq \lambda_{3}\left(P_{1}-P_{2}\right)-\gamma P_{2}, \\
& P_{2} A_{2}^{T}+A_{2} P_{2} \leq \lambda_{4}\left(P_{1}-P_{2}\right)-\gamma P_{2} .
\end{aligned}
$$

In other words, if $P_{j}$ 's and $\lambda_{p}$ 's solving the system above exist, $\dot{x}(t) \in$ $\operatorname{co}\left\{A_{1}, A_{2}\right\} x(t)$ is stable. This can be easily generalized: if (7) has a solution, then the linear differential inclusion (5) is exponentially stable (with constant $\gamma$ ), and a Lyapunov function verifying it is the max function (18). We stress that in general, existence of a solution to (7) is not necessary for stability of (5). In the case of $l=2$, by the S-procedure, the existence of a solution is necessary for (18) to be a Lyapunov function, but this is still only necessary 
for stability. For a stable LDI, while there always exists a Lyapunov function given by the pointwise maximum of quadratic functions, there may not exist one given by the maximum of two such functions.

Example 2. Consider the linear differential inclusion $\dot{x}(t) \in \operatorname{co}\left\{A_{1}, A_{2}(a)\right\}$, where

$$
A_{1}=\left[\begin{array}{cc}
-1 & -1 \\
1 & -1
\end{array}\right], \quad A_{2}(a)=\left[\begin{array}{cc}
-1 & -a \\
1 / a & -1
\end{array}\right]
$$

for $a>1$. It was used in [6] to show that the existence of a common quadratic Lyapunov function is not necessary to guarantee the exponential stability of the LDI. The maximal $a$ which ensures the existence of a common quadratic Lyapunov function was found to be $3+\sqrt{8}=5.8284$. With the phase plane method, it was confirmed that the LDI is still stable for $a=10$. However, as pointed out in [6], the analytical method is highly unlikely to be feasible for general systems.

Here we illustrate how increasing the number of matrices defining the maximum function (18) improves the estimates of parameter $a$ for which the LDI remains stable. In particular, our computation - that is, solving the system of bilinear matrix inequalities $(7)$ - carried out with 7 matrices $P_{j}$ verifies stability for $a=10.108$. The following table illustrates the maximal $a$ (denoted $a_{\max }$ ) verified by $V_{\max , l}$ given by $l=1,2, \cdots, 7$ matrices, which guarantees the stability of the LDI.

\begin{tabular}{|c|r|r|r|r|r|r|r|}
\hline $\mathrm{l}$ & 1 & 2 & 3 & 4 & 5 & 6 & 7 \\
\hline \hline$a_{\max }$ & 5.8284 & 8.109 & 8.955 & 9.428 & 9.731 & 9.948 & 10.108 \\
\hline
\end{tabular}

The 7 matrices defining $V_{\max , 7}$ that verify the stability of the LDI at $a=$ 10.108 , listed for verification, are:

$$
\begin{aligned}
& {\left[\begin{array}{rr}
0.2854 & -0.7282 \\
-0.7282 & 7.6744
\end{array}\right],\left[\begin{array}{rr}
0.5899 & -0.0010 \\
-0.0010 & 5.9677
\end{array}\right],\left[\begin{array}{rr}
0.4925 & -0.3298 \\
-0.3298 & 6.7759
\end{array}\right]}
\end{aligned}
$$

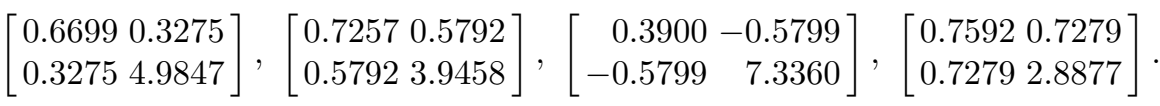

As a visual verification of stability for $a=10.108$, we sketch in Fig. 1 the vectors $A_{1} x$ and $A_{2} x$ at points $x$ on the boundary of the 1-level set of $V_{\max , 7}$; that is, points where $V_{\max , 7}(x)=1$. By linearity and homogeneity, verifying one level set is sufficient.

While Lyapunov functions, computed for example by solving (7) as described above, verify stability, they can be also used to confirm instability. We illustrate this below, where a Lyapunov function for an LDI with a certain parameter value is used to show instability when the parameter is varied.

Example 3. Recall the LDI from Example 2. In [6], it is pointed out that it may be stable for $a>10$ (while it is only verified for $a$ up to 10). Here we would like to estimate a lower bound on $a$ 's which destabilize the LDI. 

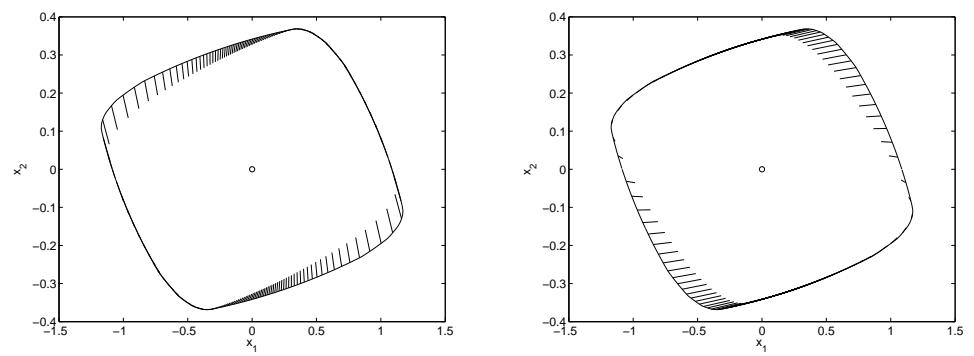

Fig. 1. $\dot{x}=A_{1} x$ (left) and $\dot{x}=A_{2} x$ (right) on the boundary of a level set

Suppose that $V_{\max }$ verifies stability of the LDI for $a$ up to $\bar{a}$. At each $x$, we find the index $i$ that maximizes $\nabla V_{\max }^{T}(x) A_{i} x$, use this to choose the "worst" switching among the vertices of the LDI, and try to produce potentially diverging trajectories for values of $a$ larger than $\bar{a}$.

Below, $V_{\max , l}$ denotes the max function given by $l$ matrices, as obtained in Example 2. By using $V_{\max , 2}$, the lower bound on $a$ 's (denoted as $\underline{a}_{\min }$ ) that guarantees instability of the LDI is detected as $\underline{a}_{\min }=12.175$. A closed limit trajectory resulting from the worst switching law at $a=12.175$ is plotted as the outer curve in the right box in Fig. 2. The inner closed curve in the same box is the boundary of the 1-level set of $V_{\max , 2}$. With $V_{\max , 7}$, the lower bound for $a$ that guarantees instability is $\underline{a}_{\min }=11.292$. The corresponding "worst" switching leads to a closed limit trajectory plotted in the middle box in Fig. 2, along with the 1-level set of $V_{\max , 7}$.

Plotted in the right box in Fig. 2 is a diverging trajectory corresponding to $l=7$ and $a=11.5$ (initial state marked with ${ }^{\prime} *^{\prime}$. As we can see from the left box and the middle box, the difference between the limit trajectory and the boundary of the level set for $l=7$ is smaller than that for $l=2$. It is expected that as $l$ is increased, the boundary of the level set can be made even closer to a limit trajectory, indicating that the Lyapunov function would give a better estimation of stability. As expected, with an increased $l$, the difference between $a_{\max }$ and $\underline{a}_{\min }$ will get smaller.
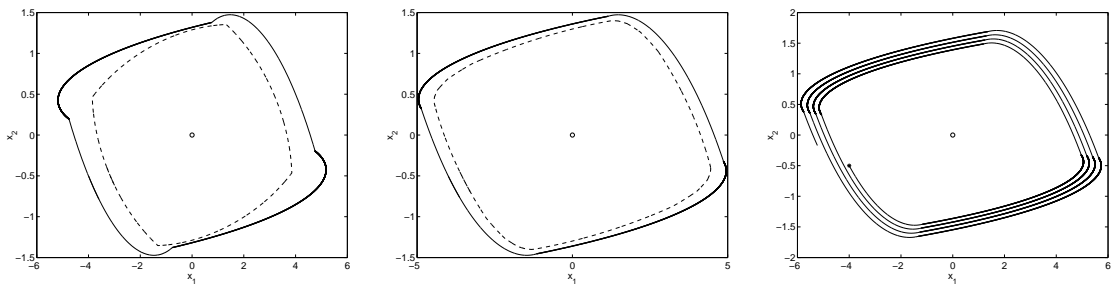

Fig. 2. Trajectories. Left: $l=2, a=12.175$; middle: $l=7, a=11.292$; left: $l=$ $7, a=11.5$ 
We now describe the conjugate of the max function (18). First, we need an additional construction. Given symmetric and positive definite matrices $Q_{1}$, $Q_{2}, . ., Q_{l}$, the minimum of the quadratic functions given by them needs not be convex. The convex hull function (determined by $Q_{j}$ 's),

$$
V_{\text {co }}(\xi)=\mathrm{co} \min _{j=1,2, . ., l} \frac{1}{2} \xi^{T} Q_{j} \xi,
$$

is the greatest convex function bounded above by the aforementioned minimum (equivalently, by each of the quadratic functions). This function is convex, differentiable, positive definite, and homogeneous of degree 2. The conjugacy relationship between the max function and the convex hull function is as follows: if $Q_{j}=P_{j}^{-1}$, then

$$
V_{\max }^{*}(\xi)=V_{\mathrm{co}}(\xi), \quad V_{\mathrm{co}}^{*}(x)=V_{\max }(x) .
$$

In other words, the conjugate of the maximum of quadratics is the convex hull of quadratics given by the inverses of the original matrices. ${ }^{8}$ If either $V_{\max }$ or $V_{\text {co }}$ is quadratic, then both of them are, and their conjugacy reduces to the conjugacy for quadratic functions as stated at the beginning of this section.

With the help of the convex hull function $V_{\text {co }}$ and the duality theory of Section 2, a condition for stability of an LDI, "dual" to matrix inequality (7), can be derived. Note that as (7) leads to stability of the linear differential inclusion (5), verified through $V_{\max }$ given by $P_{j}$ 's, the inequality (8) leads to stability of the dual linear differential inclusion (6), verified through $V_{\max }$ given by $Q_{j}$ 's. But by duality, this is equivalent to stability of (5), verified through $V_{\text {co }}$ given by $Q_{j}^{-1}$ 's. In short, existence of a solution to (8) is a sufficient condition for stability of (5). We stress again that in general, this condition is not equivalent to (7).

Just as using the same numerical test for a given LDI and its dual inclusion may result in different stability estimates (recall Example 1), solving the systems of inequalities (7) and (8) may lead to different conclusions.

Example 4. Recall the linear differential inclusion from Example 1. We used both (7) and (8), with two unknown matrices, to estimate the range of the parameter $a$ for which the LDI is stable. Using (7), we verified stability for all $a>0$. Using (8), stability is verified for $a$ up to 441 . In other words, the matrix inequality based on the max function performs better than the inequality based on the convex hull function for this particular LDI. Conversely, the convex hull function performs better than the max function for the dual LDI. See also Example 7.

Example 5. Consider the differential inclusion $\dot{x}(t) \in \operatorname{co}\left\{A_{1}, A_{2}, A_{3}\right\} x(t)$ where

\footnotetext{
${ }^{8}$ A more general relationship is valid. The conjugate of a pointwise maximum of a family of convex functions is the convex hull of their conjugates.
} 


$$
A_{1}=\left[\begin{array}{r}
-2-2 \\
4-2
\end{array}\right], \quad A_{2}=\left[\begin{array}{rr}
-1 & 0 \\
6 & -1
\end{array}\right], \quad A_{3}=\left[\begin{array}{r}
-5-3 \\
2-1
\end{array}\right] .
$$

The system is not quadratically stable, but is asymptotically (and thus exponentially) stable. It can be confirmed with the convex hull function $V_{\mathrm{co}, 2}$ given by (20) with two matrices $Q_{j}$. The convergence rate turns out to be $\gamma=0.0902$, that is

$$
\frac{d}{d t} V_{\mathrm{co}, 2}(x(t)) \leq-0.0902 V_{\mathrm{co}, 2}(x(t))
$$

for all solutions to the LDI. However, stability can not be confirmed by any of the max functions $V_{\max , 2}$ given by (18) with two matrices. The maximal $\gamma$ satisfying

$$
\frac{d}{d t} V_{\max , 2}(x(t)) \leq-\gamma V_{\max , 2}(x(t))
$$

for some $V_{\max , 2}$ is -0.1575 . Note that this in particular verifies that the LDI is not quadratically stable.

We carried out a similar experiment after doubling the dimension of the state space. Solving the bilinear matrix inequalities of Example 5 took approximately 5 to 7 seconds, solving the corresponding ones for Example 6 took approximately twice that.

Example 6. Consider $\dot{x}(t) \in \operatorname{co}\left\{A_{1}, A_{2}, A_{3}\right\} x(t)$ where

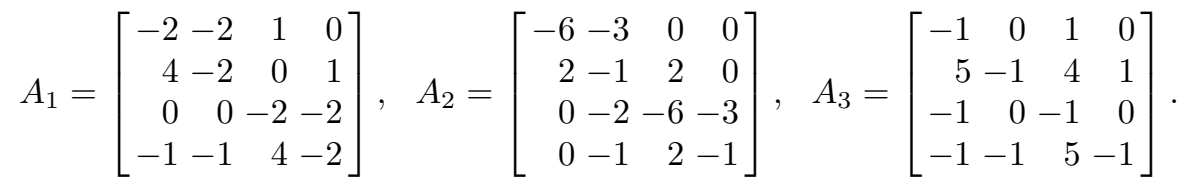

It is not quadratically stable. Stability can be confirmed with $V_{\mathrm{co}, 2}$, and the convergence rate turns out to be $\gamma=0.1948$. However, stability can not be confirmed with any $V_{\max , 2}$. The maximal $\gamma$ satisfying

$$
\frac{d}{d t} V_{\max , 2}(x(t)) \leq-\gamma V_{\max , 2}(x(t))
$$

is -0.0428 .

\section{Dissipativity properties}

The duality theory outlined in Section 2 and the tools for verifying stability as discussed in Section 3 can be extended and applied to treat dissipativity properties of linear differential inclusions with disturbances.

Consider the following LDI with external disturbance 


$$
\left[\begin{array}{l}
\dot{x}(t) \\
y(t)
\end{array}\right]=\operatorname{co}\left\{\left[\begin{array}{ll}
A & B \\
C & D
\end{array}\right]_{i}\right\}_{i=1}^{m}\left[\begin{array}{l}
x(t) \\
d(t)
\end{array}\right]
$$

It is called dissipative with (positive semidefinite) storage function $V$ and supply rate $h$ if for all $x, d$,

$$
\nabla V(x)^{T}(A x+B d) \leq h(C x+D d, d)
$$

for all

$$
\left[\begin{array}{cc}
A & B \\
C & D
\end{array}\right] \in \operatorname{co}\left\{\left[\begin{array}{cc}
A & B \\
C & D
\end{array}\right]_{i}\right\}_{i=1}^{m}
$$

For example, consider $h(c, d)=-\frac{1}{2}\|c\|^{2}+\frac{1}{2} \beta^{2}\|d\|^{2}$. Dissipativity with this supply rate means exactly that the LDI (21) has finite $L^{2}$-gain, bounded above by $\beta$. Later, we also discuss passivity and passivity with extra feedforward.

Following the ideas of Section 3, one can state sufficient conditions for dissipativity, based on considering storage functions given by a maximum of quadratic functions (18) or by a convex hull function (20). For example, if there exist symmetric and positive definite $P_{1}, P_{2}, \ldots, P_{l}$ and numbers $\lambda_{i j k} \geq 0$ for $i=1,2, \ldots, m, j, k=1,2, \ldots, l$ such that

$$
\left[\begin{array}{cc}
A_{i}^{T} P_{j}+P_{j} A_{i}+\sum_{k=1}^{l} \lambda_{i j k}\left(P_{j}-P_{k}\right)+C_{i}^{T} C_{i} & P_{j} B_{i}+C_{i}^{T} D_{i} \\
B_{i}^{T} P_{j}+D_{i}^{T} C_{i} & -\beta^{2} I+D_{i}^{T} D_{i}
\end{array}\right]<0
$$

then the LDI (21) has finite $L^{2}$-gain of at most $\beta$. Furthermore, the max function (18) is a storage function verifying this.

Example \%. Consider the LDI $\dot{x}(t) \in \operatorname{co}\left\{A_{1}, A_{2}(a)\right\}$ given by matrices (17). The LDI is stable for all $a>0$, see Example 1 or 4 . Here, we illustrate how considering the max function $V_{\max , l}$ with different $l$ yields better convergence rate estimates, for the case of $a=10000$. Furthermore, we introduce onedimensional disturbance and observation to the system, by considering $B_{1}=$ $B_{2}=\left[\begin{array}{lll}1 & 1 & 1\end{array}\right]^{T}, C_{1}=C_{2}=\left[\begin{array}{lll}1 & 1 & 1\end{array}\right]$, and $D_{1}=D_{2}=0$ in (21). For this LDI, we rely on (23) to estimate the $L^{2}$-gain. The table below shows how broadening the class of Lyapunov and storage functions improves the convergence rate and $L^{2}$-gain estimates.

\begin{tabular}{|c|c|c|c|c|c|}
\hline 1 & 1 & 2 & 3 & 4 & 5 \\
\hline \hline$\gamma$ & -1.4911 & 0.0531 & 0.1416 & 0.1642 & 0.1772 \\
\hline \hline$L^{2}$-gain & N/A & 57.6956 & 21.6832 & 18.7191 & 17.3278 \\
\hline
\end{tabular}

Duality of dissipativity properties can also be established, similarly to what we outlined for stability. Consider the dual of (21):

$$
\left[\begin{array}{l}
\dot{\xi}(t) \\
z(t)
\end{array}\right]=\operatorname{co}\left\{\left[\begin{array}{ll}
A^{T} & C^{T} \\
B^{T} & D^{T}
\end{array}\right]_{i}\right\}_{i=1}^{m}\left[\begin{array}{c}
\xi(t) \\
w(t)
\end{array}\right]
$$


Suppose that the supply rate is given by

$$
h(c, d)=\frac{1}{2}\left[\begin{array}{l}
c \\
d
\end{array}\right]^{T} M\left[\begin{array}{l}
c \\
d
\end{array}\right] \text { for } M=\left[\begin{array}{cc}
-R & Z \\
Z^{T} & S
\end{array}\right],
$$

with $R$ and $S$ symmetric, positive semidefinite and that $M$ is invertible. Note that this is the case, for example, for the supply rate corresponding to finite $L^{2}$-gain. Let the dual supply rate $h^{\sharp}$ be given by ${ }^{9}$

$$
h^{\sharp}(v, w)=-\frac{1}{2}\left[\begin{array}{c}
w \\
-v
\end{array}\right]^{T} M^{-1}\left[\begin{array}{c}
w \\
-v
\end{array}\right] .
$$

If $V$ is a convex, positive definite, and positively homogeneous of degree 2 function, then the LDI (21) is dissipative with storage function $V$ and supply rate $h$ if and only if the dual LDI (24) is dissipative with the storage function $V^{*}$ and supply rate $h^{\sharp}$, that is, for all $\xi, w$,

$$
\nabla V^{*}(\xi)^{T}\left(A^{T} \xi+C^{T} w\right) \leq h^{\sharp}\left(B^{T} \xi+D^{T} w, w\right) .
$$

A more general equivalence can be shown, where the terms $-\gamma V(x)$ and $-\gamma V^{*}(\xi)$ are added to the right-hand sides of inequalities (22) and (25). Thus, the stated equivalence generalizes the one between the stability of the LDI (5) and of its dual (6), as verified by inequalities (9), (16).

In particular, consider $h(c, d)=-\frac{1}{2}\|c\|^{2}+\frac{1}{2} \beta^{2}\|d\|^{2}$ corresponding to finite $L^{2}$-gain. Then $M=\left[\begin{array}{cc}-I & 0 \\ 0 & \beta^{2} I\end{array}\right], M^{-1}=\left[\begin{array}{cc}-I & 0 \\ 0 & \beta^{-2} I\end{array}\right]$, and $h^{\sharp}(v, w)=$ $\frac{1}{2}\|w\|^{2}-\frac{1}{2} \beta^{-2}\|v\|^{2}$. The general facts stated above imply that, for a convex, positive definite and homogeneous of degree 2 function $V$,

$$
\nabla V(x)^{T}(A x+B d) \leq-\frac{1}{2}\|C x+D d\|^{2}+\frac{1}{2} \beta^{2}\|d\|^{2}
$$

for all $x, d$ if and only if for all $\xi, w$

$$
\nabla V^{*}(\xi)^{T}\left(A^{T} \xi+C^{T} w\right) \leq-\frac{1}{2} \beta^{-2}\left\|B^{T} \xi+D^{T} w\right\|^{2}+\frac{1}{2}\|w\|^{2} .
$$

This equivalence suggests that a dual to the sufficient condition (23) for the LDI (21) to have finite $L^{2}$-gain can be stated. The dual condition relies on the convex hull function (20) serving as the storage function; and corresponds to (23) just as the dual stability condition (8) corresponds to (7). It is:

$$
\left[\begin{array}{rr}
Q_{j} A_{i}^{T}+A_{i} Q_{j}+\sum_{k=1}^{l} \lambda_{i j k}\left(Q_{j}-Q_{k}\right)+B_{i} B_{i}^{T} & Q_{j} C_{i}^{T}+B_{i} D_{i}^{T} \\
C_{i} Q_{j}+D_{i} B_{i}^{T} & -\beta^{2} I+D_{i} D_{i}^{T}
\end{array}\right]<0 .
$$

We use these dual conditions in the following example.

${ }^{9}$ Note that $h$ is a function concave in one variable, convex in the other. A conjugacy theory for such functions, extending that for convex functions, does exist. In particular, a concave/convex function conjugate to $h$ can be defined. The dual supply rate $h^{\sharp}$ is closely related to it. 
Example 8. Consider the differential inclusion

$$
\dot{x}(t) \in \operatorname{co}\left\{A_{1}, A_{2}\right\} x(t)+B d(t), \quad y(t)=C x(t),
$$

where

$$
A_{1}=\left[\begin{array}{rrr}
0 & 1 & 0 \\
0 & 0 & 1 \\
-1 & -2 & -3
\end{array}\right], \quad A_{2}=\left[\begin{array}{rrr}
0 & 1 & 0 \\
0 & 0 & 1 \\
-2 & -3 & -1
\end{array}\right], \quad B=\left[\begin{array}{r}
0 \\
-1 \\
1
\end{array}\right], \quad C=\left[\begin{array}{lll}
1 & 0 & 1
\end{array}\right] .
$$

The matrices $A_{1}$ and $A_{2}$ are taken from an example in [19].

With zero disturbance $d$, the system is stable, but not quadratically. Stability is confirmed with both the max function (18) and the convex hull function (20) with $l=2$. The convergence rate verified by $V_{\max , 2}$ and that with $V_{\mathrm{co}, 2}$ are both equal to 0.0339 , that is, (9) holds with $\gamma=0.0339$ for some max function and some convex hull function given by two quadratics.

However, the estimates of the $L^{2}$-gain of the full system, obtained by the max function and the convex hull function with two quadratics differ. They are, respectively, 16.7337 and 30.6556 .

Now consider $h(c, d)=c^{T} d-\frac{1}{2} \eta\|d\|^{2}$. Then (22) represents passivity of the inclusion with disturbance (21) when $\eta=0$, and passivity with extra feedforward when $\eta>0$. Arguments as those outlined for the $L^{2}$-gain show that for a convex, positive definite and homogeneous of degree 2 function $V$,

$$
\nabla V(x)^{T}(A x+B d) \leq(C x+D d)^{T} d-\frac{1}{2} \eta\|d\|^{2}
$$

for all $x, d$ if and only if for all $\xi, w$

$$
\nabla V^{*}(\xi)^{T}\left(A^{T} \xi+C^{T} w\right) \leq\left(B^{T} \xi+D^{T} w\right)^{T} w-\frac{1}{2} \eta\|w\|^{2} .
$$

Thus, passivity (with feedforward) of (21) is equivalent to passivity (with feedforward) of (24). Similarly one obtains that the existence of symmetric and positive definite $P_{1}, P_{2}, \ldots, P_{l}$ and numbers $\lambda_{i j k} \geq 0$ for $i=1,2, \ldots, m$, $j, k=1,2, \ldots, l$ such that

$$
\left[\begin{array}{cc}
A_{i}^{T} P_{j}+P_{j} A_{i}+\sum_{k=1}^{N} \lambda_{i j k}\left(P_{j}-P_{k}\right) & P_{j} B_{i}-C_{i}^{T} \\
B_{i}^{T} P_{j}-C_{i} & \eta-D_{i}^{T}-D_{i}
\end{array}\right]<0
$$

is a sufficient condition for passivity of (21). Of course, a dual condition can be given. We do not state it explicitly, but apply it, and (28), in examples.

Example 9. Consider (21) given by

$$
A_{1}=\left[\begin{array}{rrr}
0 & 1 & 0 \\
0 & 0 & 1 \\
-1 & -2 & -3
\end{array}\right], \quad B_{1}=\left[\begin{array}{r}
0.6 \\
0 \\
0.6
\end{array}\right], \quad C_{1}=\left[\begin{array}{ll}
0-1 & 1
\end{array}\right], \quad D_{1}=2
$$




$$
A_{2}=\left[\begin{array}{rrr}
0 & 1 & 0 \\
0 & 0 & 1 \\
-1 & -1 & -3
\end{array}\right], \quad B_{2}=B_{1}, \quad C_{2}=[0-1-1], \quad D_{2}=D_{1} .
$$

The matrices $A_{1}$ and $A_{2}$ are taken from [19], where it was shown that the system (without disturbance) is quadratically stable. Passivity of the inclusion, however, can not be confirmed with a quadratic function: the largest $\eta$ for which the passivity inequality (27) holds is -0.601 . When the maximum of two quadratic functions is used as a storage function, that is, the matrix inequality (28) is solved, the maximal $\eta$ is -0.0797 . When the convex hull function is used, the maximal $\eta$ is 0.0891 . This confirms passivity of the LDI, in fact with feedforward.

Example 10. Consider the LDI (21) with $m=2$, and with $B_{2}$ and $C_{2}$ depending on a parameter $a$. The matrices are

$$
\begin{aligned}
& A_{1}=A_{2}=\left[\begin{array}{rrr}
0 & 1 & 0 \\
0 & 0 & 1 \\
-1 & -2 & -3
\end{array}\right], \quad B_{1}=\left[\begin{array}{l}
0 \\
0 \\
1
\end{array}\right], \quad B_{2}=B_{1}+a\left[\begin{array}{r}
0 \\
-1 \\
1
\end{array}\right] \text {, } \\
& C_{1}=\left[\begin{array}{lll}
1 & 0 & 0
\end{array}\right], \quad C_{2}=C_{1}+a\left[\begin{array}{lll}
0 & 1 & 1
\end{array}\right], \quad D_{1}=D_{2}=2 \text {. }
\end{aligned}
$$

For $a=0$, the LDI reduces to a linear system, which is passive (and this can be verified with a quadratic storage function). In fact, quadratic functions verify passivity of the LDI for all $a \in[0,2.097]$. Using the maximum of two quadratic functions increases the upper limit to 2.3706 , the convex hull of two quadratics yields 2.3497 .

\section{Discrete time systems}

As we showed in Section 2, convex conjugate functions also allow for establishing a duality theory for linear difference inclusions, that is for discrete-time counterparts of the systems discussed so far. The functions proposed in Section 3 can of course also be used as Lyapunov or storage functions in discrete time, and they suggest matrix inequalities for stability and dissipativity properties.

First, consider the linear difference inclusion (14). If there exist symmetric and positive definite $P_{1}, P_{2}, \ldots, P_{l}$ and numbers $\lambda_{i j k} \geq 0$ with $\sum_{k=1}^{l} \lambda_{i j k}=1$ for $i=1,2, \ldots, m, j, k=1,2, \ldots, l$ such that

$$
A_{i}^{T} P_{j} A_{i}<\gamma \sum_{k=1}^{l} \lambda_{i j k} P_{k}
$$

for all $i=1,2, \ldots m, j=1,2, . . l$, then the discrete time Lyapunov inequality (12) holds for the max function (18), for all $A \in \operatorname{co}\left\{A_{i}\right\}_{i=1}^{m}$. Dually, if matrices $P_{j}$ and numbers $\lambda_{i j k}$ satisfy 


$$
A_{i} P_{j} A_{i}^{T}<\gamma \sum_{k=1}^{l} \lambda_{i j k} P_{k},
$$

the Lyapunov inequality (12) holds for the convex hull function (20) given by $Q_{j}=P_{j}^{-1}$. Solution of either system of matrix inequalities verifies stability of both (14) and (15).

Example 11. Consider the difference inclusion (14) given by

$$
A_{1}=\left[\begin{array}{rrr}
0.3 & 1 & 0 \\
0 & 0.6 & 1 \\
0 & 0 & 0.7
\end{array}\right], \quad A_{2}=\left[\begin{array}{rrr}
0.3 & 0 & 0 \\
-0.5 & 0.7 & 0 \\
-0.2 & -0.5 & 0.7
\end{array}\right] \text {. }
$$

The smallest $\gamma$ for which the Lyapunov inequality (12) holds with a quadratic function is 1.2353 , so a quadratic function does not verify stability of the inclusion. (For stability, we need $\gamma \in(0,1)$.) The smallest such $\gamma$ (i.e. the convergence rate) estimated with the max function with two quadratics is 0.9570 , a convex hull of two quadratics yields 1.0231 . In this case, only the max function verifies stability of the inclusion.

For linear difference inclusions with disturbance

$$
\left[\begin{array}{c}
x^{+} \\
y
\end{array}\right] \in \operatorname{co}\left\{\left[\begin{array}{ll}
A & B \\
C & D
\end{array}\right]\right\}_{i=1}^{m}\left[\begin{array}{l}
x \\
d
\end{array}\right]
$$

dissipativity properties can be verified through the following matrix inequalities. Let $\gamma>0$. If there exist symmetric, positive definite $P_{1}, P_{2}, \ldots, P_{l}$ and numbers $\lambda_{i j k} \geq 0$ with $\sum_{k=1}^{l} \lambda_{i j k}=1$ for $i=1,2, \ldots, m, j, k=1,2, \ldots, l$ such that

$$
\left[\begin{array}{cc}
A_{i}^{T} P_{j} A_{i}-\sum_{k=1}^{l} \lambda_{i j k} P_{k}+C_{i}^{T} C_{i} & A_{i}^{T} P_{j} B_{i}+C_{i}^{T} D_{i} \\
B_{i}^{T} P_{j} A_{i}+D_{i}^{T} C_{i}^{T} & -\gamma^{2} I+B_{i}^{T} P_{j} B_{i}+D_{i}^{T} D_{i}
\end{array}\right]<0
$$

for all $i=1,2, \ldots m, j=1,2, . . l$, then $\gamma$ is an upper bound for the $l^{2}$-gain of (29). This is verified by the max function $V_{\max }$ given by (18), that is, for $V=V_{\max }$ and all $x, d, x^{+}, y$ satisfying (29), the following inequality holds:

$$
V\left(x^{+}\right)-V(x)<-\frac{1}{2}\|y\|^{2}+\frac{1}{2} \gamma^{2}\|d\|^{2} .
$$

Similarly, if there exist $P_{j}$ 's and $\lambda_{i j k}$ 's such that

$$
\left[\begin{array}{cc}
A_{i}^{T} P_{j} A_{i}-\sum_{k=1}^{l} \lambda_{i j k} P_{k} & A_{i}^{T} P_{j} B_{i}-C_{i}^{T} \\
B_{i}^{T} P_{j} A_{i}-C_{i} & \eta+B_{i}^{T} P_{j} B_{i}-D_{i}^{T}-D_{i}
\end{array}\right]<0
$$

then, for $V=V_{\max }$,

$$
V\left(x^{+}\right)-V(x) \leq y^{T} d-\frac{1}{2} \eta\|d\|^{2},
$$

and thus (29) is passive (if $\eta=0$ ) and passive with feedforward (if $\eta>0$ ). Duality for discrete-time systems with disturbance can also be established, and inequalities dual to (30) and (31) can be used. 
Example 12. Consider the inclusion (29) given by

$$
\begin{gathered}
A_{1}=\left[\begin{array}{rrr}
0 & 1 & 0 \\
0 & 0 & 1 \\
0.16 & 0 & -0.32
\end{array}\right], \quad B_{1}=\left[\begin{array}{r}
2.5 \\
0 \\
0.4
\end{array}\right], \quad C_{1}=\left[\begin{array}{lll}
1 & 0 & 0
\end{array}\right], \\
A_{2}=A_{1}+\left[\begin{array}{rrr}
1 & -1 & -1 \\
1 & -3 & 0 \\
0 & -1.5 & 2
\end{array}\right] \times a, \quad B_{2}=\left[\begin{array}{r}
a \\
0 \\
2.5
\end{array}\right], \quad C_{2}=\left[\begin{array}{ll}
a-a & 1
\end{array}\right],
\end{gathered}
$$

where the parameter $a \geq 0$. The maximal $a$ ensuring stability verifiable by quadratics is 0.3153 . The maximal $a$ ensuring stability verifiable by $V_{\mathrm{co}, 2}$ or $V_{\max , 2}$ is 0.4475 . At $a=0.4475$, the convergence rate, i.e., the minimal $\beta$ such that $V\left(x^{+}\right) \leq \beta V(x)$, by quadratics is 1.3558 , while that by $V_{\mathrm{co}, 2}$ or $V_{\max , 2}$ is 1 . At $a=0.3153$, the convergence rate by quadratics is 1 , while the convergence rate by $V_{\mathrm{co}, 2}$ or $V_{\max , 2}$ is 0.6873 . With $V_{\mathrm{co}, 2}$, the $l^{2}$-gain is bounded by 7.038 , with $V_{\max , 2}$, the $l^{2}$-gain is bounded by 8.3615 .

Example 13. Consider the inclusion (29) given by

$$
\begin{aligned}
& A_{1}=\left[\begin{array}{rrr}
0 & 1 & 0 \\
0 & 0 & 1 \\
0.16 & 0 & -0.32
\end{array}\right], \quad B_{1}=\left[\begin{array}{r}
2.5 \\
0 \\
0.4
\end{array}\right], \quad C_{1}=\left[\begin{array}{lll}
1 & 0 & 0
\end{array}\right], \quad D_{1}=5, \\
& A_{2}=\left[\begin{array}{rrr}
0.4 & 0.6 & -0.4 \\
0.4 & -1.2 & 1 \\
0.16 & -0.6 & 0.48
\end{array}\right], \quad B_{2}=\left[\begin{array}{r}
0.4 \\
0 \\
2.5
\end{array}\right], \quad C_{2}=\left[\begin{array}{ll}
0.4-0.41
\end{array}\right], \quad D_{2}=6,
\end{aligned}
$$

Stability is not confirmed by quadratics while $V_{\mathrm{co}, 2}$ and $V_{\mathrm{max}, 2}$ both ensure a convergence rate of 0.7530 . With $V_{\max , 2}$, the maximal $\eta$ such that (32) is -0.0148 . With $V_{\mathrm{co}, 2}$, the maximal $\eta$ is 0.5078 .

In the concluding example, we outline an application of the dual BMIs technology to estimation of the domain of attraction of a nonlinear system.

Example 14. Consider a second order saturated linear system

$$
x^{+}=A x+B \operatorname{sat}(F x),
$$

where

$$
A=\left[\begin{array}{rr}
0.8 & 0 \\
0 & 1.2
\end{array}\right], \quad B=\left[\begin{array}{l}
0.6 \\
-1
\end{array}\right], \quad F=\left[\begin{array}{ll}
0.5 & 1
\end{array}\right],
$$

and sat is the standard saturation function, that is $\operatorname{sat}(s)=s$ when $s \in[-1,1]$ and $\operatorname{sat}(s)=1$ (respectively, -1 ) when $s>1$ (respectively, $s<-1$ ). We want to estimate the domain of attraction of this system with contractively invariant 1-level set of certain type of Lyapunov functions. For a given $x_{0}$, the 
Lyapunov function is optimized so that its invariant level set contains $\alpha x_{0}$ with a maximal $\alpha$.

We first consider quadratic functions. Using the algorithm from [12], the maximal $\alpha$ for $x_{01}=\left[\begin{array}{ll}1 & 0\end{array}\right]^{T}$ is $\alpha_{1}=5.0581$ and the maximal $\alpha$ for $x_{02}=$ $\left[\begin{array}{ll}0 & 1\end{array}\right]^{T}$ is $\alpha_{2}=3.6402$.

Next we consider $V_{\mathrm{co}, 2}$. An algorithm to maximize $\alpha$ so that $\alpha x_{0}$ is inside an invariant level set of $V_{\mathrm{co}, 2}$ can be developed similarly to that in Section 3 of [13]. From this algorithm, the maximal $\alpha$ for $x_{01}$ is $\tilde{\alpha}_{1}=7.1457$ and the maximal $\alpha$ for $x_{02}$ is $\tilde{\alpha}_{2}=5$. In the left box of Fig. 3, we plot the level set (thick solid curve) as the convex hull of two ellipsoids (dash-dotted line). The point on the thick curve marked with " $*$ " is $\tilde{\alpha}_{2} x_{02}$. The inner ellipsoid in solid line is the maximal invariant ellipsoid where the point marked with " $*$ " is $\alpha_{2} x_{02}$. To demonstrate that the level set is actually invariant, we plot the image of the boundary under the next step map $x \mapsto A x+B$ sat $(F x)$ (see the thin solid curve in the right box of Fig. 3). For comparison, we also plot the image under the linear map $x \mapsto(A+B F) x$ in dashed curve. Parts of the thin solid curve overlap with the thick solid curve. This means that some trajectories overlap parts of the boundary of the level set. As a matter of fact, for any $\alpha>\tilde{\alpha}_{2}$, a trajectory starting from $\alpha\left[\begin{array}{ll}0 & 1\end{array}\right]^{T}$ will diverge.
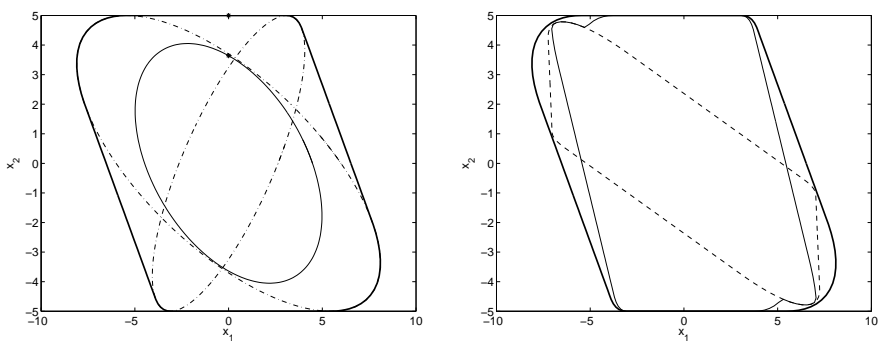

Fig. 3. Left: the invariant level set; right: the next step map.

Acknowledgment. Research by T. Hu and A.R. Teel was supported in part by the ARO under Grant no. DAAD19-03-1-0144, the NSF under Grant no. ECS-0324679, and by the AFOSR under Grant no. F49620-03-1-0203.

\section{References}

1. Beran E, Vandenberghe L, Boyd S (1997) A global BMI algorithm based on the generalized Benders decomposition In: Proc. of the European Control Conference paper no. 934

2. Blanchini F (1995) Nonquadratic Lyapunov functions for robust control. Automatica, 31:451-461 
3. Boyd S, Ghaoui L. E, Feron E, Balakrishnan V (1994) Linear Matrix Inequalities in System and Control Theory. SIAM

4. Brayton R, Tong C (1979) Stability of dynamical systems: a constructive approach. IEEE Trans. on Circuits and Systems 26:224-234

5. Chesi G, Garulli A, Tesi A, Vicino A (2003) Homogeneous Lyapunov functions for systems with structured uncertainties. Automatica 39:1027-1035

6. Dayawansa W, Martin C (1999) A converse Lyapunov theorem for a class of dynamical systems which undergo switching. IEEE Trans. Automat. Control 44(4):751-760

7. Goebel R, Teel A, Hu T, Lin Z (2004) Dissipativity for dual linear differential inclusions through conjugate storage functions In: Proc. of the 43rd IEEE Conference on Decision and Control, Bahamas

8. Goh K, Safonov M, Papavassilopoulos G. P (1994) A global optimization approach for the BMI problem p. 2009-2014., In: Proc. of the 33rd IEEE Conference on Decision and Control

9. Hassibi A, How J, Boyd S (1999) A path-following method for solving BMI problems in control p. 1385-1389., In: Proc. of American Control Conference

10. Hu T, Huang B, Lin Z (2004) Absolute stability with a generalized sector condition. IEEE Trans. Automat. Contr., 49(4):535-548

11. Hu T, Lin Z (2003) Composite quadratic Lyapunov functions for constrained control systems. IEEE Trans. Automat. Contr., 48(3):440-450

12. Hu T, Lin Z, Chen B (2002) Analysis and design for linear discrete-time systems subject to actuator saturation. Systems \& Control Lett., 45(2):97-112

13. Hu T, Lin Z, Goebel R, Teel A (2004) Stability regions for saturated linear systems via conjugate Lyapunov functions In: Proceedings of the 43rd IEEE Conference on Decision and Control, Bahamas

14. Jarvis-Wloszek Z, Packard A (2002) An LMI method to demonstrate simultaneous stability using non-quadratic polynomial Lyapunov functions p. 287-292., In: Proc. 41st IEEE Conf. Decision and Control, Las Vegas, 2002

15. Molchanov A, Pyatnitskiy Y (1989) Criteria of asymptotic stability of differential and difference inclusions endountered in control theory. System Control Lett., 13:59-64

16. Power H, Tsoi A (1973) Improving the predictions of the circle criterion by combining quadratic forms. IEEE Trans. Automat. Control, 18(1):65-67

17. Rockafellar R (1970) Convex Analysis. Princeton University Press

18. Rockafellar R, Wets R. J.-B (1998) Variational Analysis. Springer

19. Shorten R. N, Narendra K. S (2003) On common quadratic Lyapunov functions for pairs of stable LTI systems whose system matrices are in companion form. IEEE Trans. Automat. Control 48(4):618-621

20. Willems J (1972a) Dissipative dynamical systems, part I: General theory. Arch. Rational Mech. Anal. 45:321-351

21. Willems J (1972b) Dissipative dynamical systems, part II: Linear systems with quadratic supply rates. Arch. Rational Mech. Anal. 45:352-393

22. Xie L, Shishkin S, Fu M (1997) Piecewise Lyapunov functions for robust stability of linear time-varying systems. Systems \& Control Lett. 31:165-171

23. Zelentsovsky A (1994) Non-quadratic Lyapunov functions for robust stability analysis of linear uncertain systems. IEEE Trans. Automat. Control 39(1):135138 